\title{
Alcohol y violencia familiar
}

\author{
García MÁs, Mà.P. \\ Socióloga \\ Enviar correspondencia a: \\ Mary-Pepa García Más. INSS. Padre Damián, 4 - 5ª planta. 28036 MADRID
}

\section{RESUMEN}

La violencia doméstica es un fenómeno que afecta a un número cada vez más elevado de mujeres en nuestro país, debido a la influencia de los patrones socioculturales más tradicionales y a prejuicios basados en la desigualdad entre los sexos. Las actitudes de tolerancia hacia los malos tratos en el hogar sin distinciones relativas al estatus socioeconómico, nivel de formación, cohortes de edad y estado civil tienen como resultado un daño de naturaleza física, sexual y psicológica. Este análisis se centra en las distintas acciones llevadas a cabo por las administraciones públicas y las organizaciones no gubernamentales respecto a la violencia y el alcohol en nuestro país y en la Unión Europea. En ellas se destaca la falta de políticas definidas sobre esta problemática, al dedicar una mayor atención a las repercusiones de las drogas ilegales en detrimento de una concienciación de la opinión pública sobre el peligro que supone la educación y el cuidado de los hijos en estado de embriaguez y sus repercusiones en la violencia. Se constata la falta de aportación y, por tanto, el desconocimiento de los datos referidos a la incidencia del alcohol en las publicaciones sobre violencia familiar en los casos en que hubiera actuado como factor desencadenante. Se concluye con las medidas a tomar frente al consumo de alcohol y violencia.

Palabras clave: alcohol, genero, violencia familiar, prevención.

\section{ABSTRACT}

Domestic violence is a social phenomenon that affects to an increasing number of women in Spain, due to the influence of the most traditional socio-cultural patterns and prejudices based on the disparity between genders. It exists a general tolerance attitude regarding domestic violence against women, with no distinctions of the socio-economic status, education level, age cohorts or marital status that give as a result a physical, sexual and psychological damage. The analysis is focused on the different actions carried out by public administrations and non-governmental organisations regarding violence and alcohol both in Spain and the European Union. It is highlighted the lack of clear policies about problems related to alcohol, as they focus instead on the impact of illegal drugs, acting carelessly when raising consciousness to the public opinion about the danger that means the education and care of children when being inebriated and its impacts in violence. It is stated the lack of contribution and, therefore, of ignorance of the data referred to the incidence of alcohol in publications about domestic violence when it would have acted as an unchaining factor. The research finishes with the measures that need to be taken against alcohol and violence.

Key words: alcohol, gender, domestic violence, prevention.
$\mathbf{L}$ as sociedades industriales presentan fenómenos que ponen de manifiesto la conflictividad entre el sujeto y la sociedad como son una serie de conductas desviadas entre las que destacamos el abuso del consumo de sustancias tóxicas y las farmacodependencias. El alcohol ocupa un lugar preferente entre éstas sustancias sirviendo en muchos casos como excusa para cometer delitos contra las personas, por la incapacidad de algunos varones para hacer frente a los problemas esencialmente económicos y laborales, sin recurrir al alcohol. Tanto en la familia como en el trabajo o incluso en el ámbito social, existen agresiones físicas y psíquicas derivadas no sólo del desequili- brio de las relaciones entre los sexos sino también de las influencias históricas y culturales que mantienen actitudes de tolerancia hacia los malos tratos en contra de las mujeres.

El número de muertes de mujeres en manos de sus agresores ha aumentado en mayor medida que las denuncias presentadas durante el periodo de aplicación del I Plan de Acción contra la Violencia Doméstica. En el año 2000 fallecieron 44 mujeres, lo que supone un aumento del $25 \%$ respecto a 1998 frente a un incremento del $14 \%$ de denuncias de víctimas de malos tratos entre 1998 y 1999. En noviembre de 2001, las denuncias por malos tratos superaron las 
veinte mil entre enero y octubre. Sólo el 4,2\% de las mujeres españolas mayores de edad reconocen haber sufrido malos tratos aunque el porcentaje real de víctimas de este delito alcanza el 12,4\%.

A nivel internacional, según datos obtenidos de la "Conferencia Internacional sobre la Violencia Contra las Mujeres" Ilevada a cabo en Estocolmo en abril de 2001 sobre los índices de violencia en distintos países, en Suecia una mujer es acosada cada 24 horas, y entre 20 y 30 mujeres mueren al año a manos de sus cónyuges o compañeros, mientras que en Finlandia, el $22 \%$ de las mujeres casadas son maltratadas por sus parejas. En Gran Bretaña, una mujer muere cada tres días víctima de conductas violentas. En ninguno de los datos publicados relacionados con los malos tratos o muertes de mujeres maltratadas, y los referidos a las denuncias presentadas, se especifica los casos en los que el alcohol pudiera haber actuado como factor desencadenante en el acto de agresión.

El II Plan de Acción contra la Violencia Doméstica estará vigente hasta el año 2004, con la colaboración de cinco ministerios, las Comunidades Autónomas, las corporaciones locales y las ONG dedicadas a estos temas. Este plan en realidad se centra en mayor medida en la asistencia social aunque influyan las posibles reformas legislativas favorables a las mujeres maltratadas que hubieran denunciado. No obstante, una vez más, no aparecen referencias específicas de los agresores cuyos abusos físicos y psicológicos tienen como base un excesivo consumo de bebidas alcohólicas, ni en el ámbito jurídico existen referencias a sentencias en juicios por malos tratos en los que esté presente una intoxicación etílica habitual, ocasional o crónica, a excepción de las analizadas en los procedimientos judiciales.

Son numerosos los autores que consideran de suma importancia los conflictos de índole social en el incremento del índice de malos tratos. En opinión de Santiago Delgado (1), "cuando un hecho violento ocurre en el seno de la sociedad, significa que, de alguna manera, se está produciendo una fuga en los mecanismos de control social de las conductas, clasificando la conducta violenta, dentro de la patología reaccional, como una reacción primitiva de carácter impulsivo, típica de psicóticos e intoxicados". A pesar de ello, es asumido de forma unánime, que toda conducta violenta no puede reducirse a la existencia de un trastorno mental o consumo de bebidas alcohólicas. Este autor otorga una enorme trascendencia a la falta de un enfoque definido de las autoridades responsables del área de la salud sobre la creación de programas dirigidos a hacer frente a los problemas relacionados con el alcohol.

Por tanto, a pesar de los avances conseguidos, todavía se hace necesario un mayor esfuerzo por parte de los agentes sociales. De hecho, que la situa- ción haya mejorado, ha fomentado no sólo la sensibilización de la opinión pública sino también el aumento de mujeres que se atrevan a denunciar públicamente la vergüenza de la vejación de la que han sido víctimas. En multitud de casos el silencio se ha debido a la errónea confianza de lograr abordar y solucionar el conflicto sin la imprescindible ayuda externa.

En esta problemática cobra vital relevancia la cooperación de la sociedad civil a través de las ONG, particularmente, las especializadas en mujeres y alcoholismo.

Respecto a este último tema llamamos la atención sobre la importancia de las asociaciones de autoayuda. Pioneros de estos movimientos fueron los ex-alcohólicos anónimos en los años 20 del siglo pasado, que hicieron posible la aparición de las conocidas asociaciones de ex-alcohólicos rehabilitados que forman parte del tejido social propio de las sociedades más desarrolladas. Estas organizaciones plantearon aspectos novedosos en el momento de su creación como la tipificación del alcoholismo como una enfermedad y sus repercusiones en el individuo y su medio, así como la consideración de que la conducta adictiva no era voluntaria sino que partía de mecanismos inherentes al consumo de esa sustancia. Estos se relacionaban con factores de riesgo de carácter social, económico y laboral y provocaban alteraciones psicopatológicas, conductuales, sociales y sanitarias. Y lo que es más importante, iniciaron un nuevo concepto de autoayuda en la prevención de las recaídas así como la adopción de un enfoque terapéutico de su etapa rehabilitadora que tendría como objetivo último, el reforzamiento de la responsabilidad individual de cada miembro de estas asociaciones y de la población diana en la que se va a actuar en materia preventiva.

Sus objetivos y fines, sin embargo, han facilitado en muchos casos, argumentos en los que se han apoyado sentencias injustas pronunciadas por los jueces en casos de malos tratos, particularmente, en los que se da una relación directa entre la violencia familiar y el consumo excesivo de alcohol. Por otra parte, la consideración del alcoholismo como una enfermedad, es uno de los motivos que ha dificultado o retrasado la denuncia o la ruptura matrimonial por parte de las mujeres que acuden a estas asociaciones, unido al hecho de que sus parejas estuvieran bajo tratamiento bajo tratamiento o hubieran dejado de beber. De manera que se intensifica una tendencia en este colectivo de mujeres, que sostiene la creencia de que la causa de la violencia es el alcoholismo y, consecuentemente, dejará de producirse con la desaparición de éste.

Todos estos aspectos recogen los factores de riesgo que determinan conductas agresivas relacionadas con el alcohol y la necesidad de un mayor número de centros de tratamiento y terapias ocupacionales, muy 
estrechamente relacionada con la problemática familiar derivada de la ingesta de bebidas alcohólicas.

Las conclusiones más importantes del informe realizado por la Organización para la Cooperación y Desarrollo Económico OCDE (2) sobre los "Problemas en la Familia", indican que a pesar de que en los países del marco de esta organización en los años sesenta y setenta del siglo pasado se observó un aumento del consumo de alcohol éste descendió posteriormente en las dos décadas siguientes. El tabaco y el alcohol, están registrados por la OCDE como factores causantes de una mayor incidencia en casos de muerte prematura, y de desajustes familiares y violencia. Los datos de los españoles en estos últimos años muestran un consumo de 11,9 litros por habitante mayor de 15 años. La probabilidad de sufrir dichos problemas crece con el aumento de la cantidad consumida de alcohol y con la frecuencia de intoxicación.

Los datos referidos a la "Encuesta Domiciliaria sobre consumo de Drogas" (3) realizada por el Plan Nacional sobre Drogas en 1997, llamaba la atención sobre la existencia de un fenómeno emergente de gran relevancia sociológica y epidemiológica, al reflejarse por primera vez el consumo de alcohol entre mujeres de 15 a 18 años, con porcentajes ligeramente superiores a los varones de su mismo grupo de edad para el indicador de consumo en el último año. Este punto hizo prever que la proporción igualitaria de consumo entre ambos sexos bien podía provocar un nuevo ciclo expansivo de alcohol en nuestro país, al tener en cuenta que la igualdad de consumo entre los sexos se produce en los segmentos de edad más jóvenes y en algunas modalidades de consumo de alcohol en el último año aunque de una manera menos problemática pues al descender los índices de consumo diario se reducía también en un $2 \%$ entre 1995 y 1997. Comparada con la "Encuesta Domiciliaria sobre consumo de Drogas" (4) de 1999, se observa que a pesar de una progresiva reducción de la edad de inicio y de presencia femenina, sigue detectándose un importante crecimiento del consumo de alcohol en mujeres entre 15 y 19 años. Por otra parte, se detecta una reducción en el número de consumidores abusivos de algunas drogas. Esta disminución es especialmente relevante entre los que además son consumidores de alcohol, cuyo número en los casos de bebedores de gran riesgo en días laborales, se ha reducido a un tercio del índice alcanzado en 1997. El consumo diario de alcohol es 3,4 veces superior entre los hombres que en las mujeres, lo que diferencia este indicador de los anteriores, consolidándose como un patrón esencialmente masculino.

En relación a estos datos, tanto a nivel nacional como europeo, cobra especial relevancia el informe sobre los "Problemas con el Alcohol en la Familia" (2), financiado por la Comisión Europea y realizado por un grupo de trabajo compuesto por representantes de todos los estados miembros de la Unión Europea y Noruega, denominados EUROCARE y COFACE. En él se muestra cómo a pesar de confirmarse que los problemas derivados del consumo de bebidas alcohólicas afecta a millones de familias de la Unión Europea con repercusiones negativas mayores y más graves que las drogas ilegales como pueden ser la violencia y el divorcio, los gobiernos prestan menor atención a su prevención y no conceden la suficiente importancia a los desajustes familiares producidos por el alcohol, ni dedican los recursos necesarios para una adecuada información, pues ni siquiera aparecen reflejados en las estadísticas oficiales. En este sentido, el informe llama la atención sobre la relevancia de las campañas de concienciación de los ciudadanos llevadas a cabo en todos los países estudiados sobre el peligro de la conducción en estado de embriaguez, descuidando la prevención del peligro que supone ocuparse de la educación y cuidado de los hijos en episodios de intoxicación etílica y todavía más si cabe, su incidencia en la violencia contra las mujeres. En España en 1999, el $34,36 \%$ de los recursos de la justicia en materia penal se dedicaron a sancionar conductores ebrios.

Destaca el hecho de que "la mayoría de las personas que tienen este problema lo sufren en silencio". En este orden de cosas, se pone de manifiesto que cuando "el problema se produce tras el consumo de esas sustancias, se observa una tendencia generalizada a culpar a las drogas; al contrario que cuando se produce por consumo de alcohol, siendo al bebedor, al que se le considera culpable", de este modo se distrae la atención sobre los factores sociales y económicos que fomentan el consumo problemático de bebidas alcohólicas. Es decir, admitir la influencia de estos factores forzaría indefectiblemente a tener que tomar medidas en ese sentido así como en el tema de la violencia contra las mujeres.

Según el mencionado informe, los niños y el resto de los miembros familiares del alcohólico, son víctimas de la falta de coordinación existente entre unos y otros centros de asistencia. Además ésta suele prestarse cuando el alcohólico se encuentra en tratamiento, en caso contrario, en la mayoría de ocasiones la familia no recibe el apoyo de ningún especialista.

El Plan Europeo de Actuación sobre el Alcohol 2000-2005 (PEAA) (5), fue ratificado por el Comité Regional para Europa de la Organización Mundial de la Salud celebrado en 1999 en Italia sirviendo como base para el desarrollo y la implantación de las políticas y programas en materia de alcohol en los estados miembros. Sus objetivos globales tratan entre otros de la necesidad de reducir tanto la extensión como la gravedad del daño relacionado con el alcohol en toda la Región Europea. También asocia el consumo de alcohol y la frecuencia de consumos excesivos a un mayor riesgo de casos de muertes y accidentes, incluidos los accidentes de tráfico y la violencia delibe- 
rada, tanto hacia sí mismo como hacia los demás así como el suicidio, los malos tratos, las crisis familiares y comportamientos delictivos tales como el robo y la violación.

Por su parte, la Carta Europea sobre Alcohol contemplada en el Plan Europeo de Actuación sobre el Alcohol (5), cuyas directrices se dirigen a reducir el daño que directa o indirectamente produce el alcohol, establece cinco principios éticos y diez estrategias de acción entre los que destacamos los que señalan que todas las personas tienen derecho a que su familia, comunidad y vida laboral, estén protegidos de accidentes, violencia familiar u otras consecuencias negativas asociadas al consumo de alcohol; o el que indica que todos los niños y adolescentes tienen derecho a crecer en un ambiente protegido de las consecuencias negativas asociadas a su consumo. En cuanto a las estrategias y en relación a las organizaciones no gubernamentales, recomienda apoyarlas así como a los movimientos de autoayuda que promueven iniciativas dirigidas a la prevención o reducción del daño que puede ocasionar el alcohol.

La Oficina Regional de la OMS para Europa (PEAA) (5) ofrece un marco actualizado para la Región Europea. Como apoyo activo para su implantación se creó SALUD21 encargado de señalar acciones de la Oficina Regional en cinco áreas y actuar como "conciencia sanitaria; siendo el centro de información sobre cuestiones relacionadas con el alcohol; analizando y abogando por el PEAA, ofreciendo orientación y apoyo en materia de desarrollo e implantación de políticas sobre el alcohol a los estados miembros; promocionando herramientas y directrices basadas en la experiencia y destinadas a hacer factibles las políticas sobre el alcohol; trabajando como catalizador de las acciones, ofreciendo apoyo técnico y liderazgo en los esfuerzos dirigidos a eliminar o controlar los efectos del consumo de bebidas alcohólicas".

En relación con los principios y estrategias marcados por la Carta Europea y el Plan de Actuación sobre el Alcohol (6), recomienda que cada uno de los estados miembros considere en primera instancia la naturaleza de los problemas relacionados con el alcohol y determine cuáles de las posibles acciones enumeradas serán las más aplicables y eficaces en sus circunstancias particulares, debido a que no existe un único modelo individual que pueda aplicarse en toda la Región Europea. Lo más importante es que los estados miembros adopten las acciones que ofrezcan las mayores probabilidades de reducir el peligro que representa el alcohol en sus respectivos países.

El Ministerio del Interior a través de la Delegación del Gobierno para el Plan Nacional sobre Drogas se encarga de cumplir los compromisos internacionales adquiridos por nuestro país en la XX Asamblea Extraordinaria de la Asamblea General de las Naciones Uni- das celebrada en junio de 1998. En ella se aprobó la solicitud de la elaboración de estrategias nacionales por parte de todos los estados miembros para conseguir una serie de objetivos para el año 2003 y el 2008 sobre drogodependencias basados fundamentalmente en la prevención de los consumos y sus consecuencias teniendo en cuenta las transformaciones que ha experimentado este fenómeno en los últimos tiempos. Para ello se han puesto en marcha medidas preventivas destinadas a frenar el consumo de alcohol mediante campañas de sensibilización de la opinión pública, la familia, la escuela y el mundo laboral así como otros programas de reducción de daños, asistencia e integración social sin olvidar la investigación y la formación. En este último campo, el Instituto Nacional de Investigación y Formación de Drogas nace motivado por el deseo de impulsar y mejorar la calidad de los programas en esta materia de igual manera que la puesta en marcha de una red autonómica de puntos focales de documentación provista de nuevas tecnologías de acceso a la información.

Entre los objetivos asignados al Observatorio Español de Drogas, se incluye el brindar una visión global, fiable, permanente y actualizada de los problemas relacionados con los consumos de drogas, resaltando por su especial importancia la síntesis de los resultados de la Encuesta Domiciliaria sobre Drogas. Entre sus actuaciones se encuentra el haber arbitrado el diseño de un sistema de evaluación que permite la detección precoz de las desviaciones en los objetivos marcados.

No hay que olvidar que existen múltiples asociaciones entre el consumo de alcohol y la agresividad. Precisamente teniendo en cuenta la naturaleza de éstas, entre las que destaca el miedo, los antecedentes biológicos, las experiencias infantiles de conductas violentas en la familia de origen en la figura de la madre así como las diversas vías que pueden ser empleadas para su consumo cada vez se hace más necesario desarrollar medidas efectivas para prevenir la violencia relacionada con el alcohol, ya que a pesar de los logros de la ciencia dirigidos a aportar soluciones al problema como son un mayor conocimiento sobre el uso abusivo de alcohol y el alcoholismo, no se ha llegado a una total comprensión de las causas que originan la violencia.

La investigación de carácter sociológico puede contribuir a un mayor conocimiento sobre los individuos con problemas de alto riesgo por su excesivo consumo de alcohol y continuas conductas violentas, en función del contexto ambiental donde se encuentren ubicados, los patrones socioculturales y el proceso de socialización recibido en su ámbito familiar. De esta manera se incrementa la posibilidad de llevar a cabo intervenciones efectivas desde un punto de vista social y sanitario contribuyendo así a la puesta en 
práctica de medidas preventivas frente a los propios agresores y la sociedad.

Socidrogalcohol, asumiendo las directrices marcadas a nivel internacional a través de la XX Asamblea Extraordinaria de la Asamblea General de las Naciones Unidas para el periodo del año 2000 al 2008 y consciente de la existencia de una estrecha relación entre violencia familiar y abuso/dependencia alcohólica, solicitó una subvención al Plan Nacional sobre Drogas para la realización de una investigación de carácter empírico descriptivo denominado "Evaluación e intervención de la violencia familiar y alcoholismo" (6). El objeto de estudio fueron dos colectivos de mujeres, las solicitantes de ingreso en un Centro de Acogida para víctimas de malos tratos y las asistentes a las asociaciones de ex-alcohólicos rehabilitados que aceptaron participar voluntariamente en el estudio, llevado a cabo en zonas geográficas de alta densidad de población como Madrid, Barcelona, Córdoba y Comunidad Valenciana (Castellón, Valencia y Alicante). En la investigación mencionada (6), se pudo ratificar que aunque el alcohol no constituye el único factor de riesgo que provoca la violencia familiar, un número elevado de entrevistadas de ambos colectivos, atribuyen a éste la causa principal de la violencia sufrida debido a la alta dependencia etílica de sus cónyuges o compañeros. A nivel general, destacan porcentajes significativos de consumidores habituales con una frecuencia de tres o más veces a la semana, un descenso de consumidores semanales o de fin de semana y casi la práctica desaparición de los consumidores diarios $u$ ocasionales, con un índice de continuidad de consumo de más de un año, o de uno a seis meses.

Paralelamente organizó un simposium científico sobre "Alcohol y Violencia Doméstica" con el objetivo de aportar un mayor conocimiento sobre dicha problemática e intercambiar experiencias y estudiar diferentes proyectos relativos a las consecuencias de los malos tratos en el ámbito privado del hogar frente a los diferentes factores de riesgo, entre los que ocupa un lugar primordial el uso abusivo de bebidas alcohólicas.

Socidrogalcohol se plantea seguir realizando actividades profesionales e investigaciones de carácter sociológico y epidemiológico, así como análisis cualitativos sobre la influencia del alcohol en los problemas familiares con el objeto de dar a conocer en todo su alcance los hábitos de consumo excesivo de alcohol en los individuos considerados como población de alto riesgo y su incidencia en los casos de violencia en el ámbito privado del hogar. Con ello, pretende seguir una línea de actuación comprometida y colaboradora con la problemática del alcoholismo, potenciando la puesta en práctica de nuevas políticas asistenciales y medidas efectivas para prevenir la violencia relacionada con la ingesta alcohólica.
Según datos del "Barómetro marzo 2001" (7), realizado por el Centro de Investigación Sociológicas (CIS), pendiente de su publicación, en el que se incorporan preguntas acerca de la violencia doméstica, entre las causas que llevan a ejercer la violencia, el 95,8\% de los españoles señala el abuso del alcohol, seguido a muy corta distancia por el consumo de drogas con un $94,7 \%$, los problemas psicológicos y mentales obtienen un $90,7 \%$ y el paro un $68,2 \%$. El $83,2 \%$ considera determinante que el agresor haya sido a su vez en el pasado víctima de malos tratos y abusos tanto físicos como sexuales.

Las exigencias dirigidas a la consecución de una mayor seguridad de las mujeres víctimas de malos tratos indicadas por el Ministerio de Trabajo y Asuntos Sociales en su II Plan de Actuación contra la Violencia Doméstica, son un fiel reflejo de los datos obtenidos sobre las actitudes y opiniones de ambos colectivos de mujeres (las asistentes a centros de acogida y a asociaciones de ex-alcohólicos), poniéndose de relieve su preocupación por la lentitud de la justicia. Este punto demuestra la necesidad de solicitar la incapacidad del marido o compañero para conservar la custodia de los hijos además de la obligatoriedad de seguir un tratamiento de deshabituación alcohólica o psiquiátrico, por los problemas mentales derivados de un consumo abusivo de bebidas alcohólicas, especialmente las pertenecientes a los cohortes de edad comprendidos entre los 20 y los 35 años y las mayores de 45 años.

Estos datos ofrecen una visión general suficientemente definida que permite afirmar que el tema de la violencia doméstica relacionada con un consumo excesivo de alcohol no ha sido desarrollado en todas sus vertientes. Por ello habría que analizar con mayor profundidad las causas objetivas del alcohol así como hasta qué punto produce un efecto desinhibidor de una situación de violencia y bajo qué condiciones actúa como factor de riesgo, es decir, hasta qué punto provoca situaciones de violencia, al haberse comprobado el elevado desconocimiento que muchas mujeres tienen de las situaciones de violencia en las que se encuentran inmersas, debido a la habituación a las conductas violentas, pudiendo ser el alcohol una causa desencadenante más importante de lo que en principio pueda parecer.

\section{MEDIDAS GENERALES}

La revisión de los distintos informes e investigaciones en los países de la Unión Europea sugiere una serie de medidas descritas a continuación.

Definir las consecuencias del alcohol en la problemática familiar y los malos tratos, especificando en las 
estadísticas oficiales y en aquellos datos publicados sobre la violencia doméstica, los casos en los que el consumo de alcohol haya actuado como factor desencadenante.

Arbitrar una política nacional de salud pública relacionada con el alcohol y la violencia familiar armonizada con el Plan Europeo de Actuación sobre el Alcohol y la Carta Europea de Derechos Fundamentales.

Fomentar la investigación y el control sobre el abuso de bebidas alcohólicas por parte de los gobiernos con el fin de facilitar la obtención de la información más exacta y veraz sobre la influencia del alcohol como la falta de atención y el maltrato a los hijos y la violencia doméstica entre cónyuges.

Promover campañas de información relativas al daño que puede ocasionar el alcohol y sus consecuencias en la violencia doméstica, la salud y el bienestar de los individuos y la comunidad, a través de una concienciación de la opinión pública y de los medios de comunicación social.

Garantizar que la educación para la salud en materia de alcohol y violencia doméstica en el medio escolar, erradique los sistemas de valores tradicionales perniciosos y los patrones socioculturales sobre las desigualdades por género.

Facilitar la formación de los profesionales en el ámbito jurídico, sanitario y de los servicios sociales sobre la violencia familiar y el consumo de alcohol.

Lograr en las políticas nacionales un enfoque coordinado que incluya todos los campos relacionados con esta problemática como la acogida, la asistencia médica, los servicios sociales, el ámbito jurídico y los grupos de autoayuda, en los casos en los que el alcohol se relacione con las conductas agresivas de sus cónyuges o compañeros.

Obtener una referencia explícita a los problemas familiares derivados del consumo excesivo de alcohol en las diferentes políticas nacionales mediante la solicitud a los servicios sanitarios locales y a las autorida- des responsables, de la creación de centros de asistencia para hacer frente a los problemas relacionados con el alcohol que permitan conocer las necesidades de todos los miembros de la familia, incluidas las mujeres drogadictas con cargas familiares.

Designar un miembro del equipo en las organizaciones no gubernamentales especializadas en el tratamiento de los problemas del alcohol que se responsabilice de la asistencia a la familia y a los hijos.

\section{BIBLIOGRAFÍA}

(1). Delgado Bueno, S. Aspectos psiquiátricos de la violación: lesiones psíquicas en las víctimas de violación. En: Psiquiatría Legal y Forense. Editorial Colex; 1994. p. 239-314.

(2). "Problemas con el alcohol en la familia: Un Informe para la Unión Europea". Comisión Europea. Luxemburgo. En: McNeill, A. Alcohol en la familia. Revista Adicciones. 11 (1): p. 3-6.

(3). "Encuesta Domiciliaria sobre Consumo de Drogas. 19951997". Delegación del Gobierno para el Plan Nacional sobre Drogas. Ministerio del Interior. Observatorio Español sobre Drogas. Informe n 2. 1998.

(4). "Encuesta Domiciliaria sobre Consumo de Drogas. 1999". Delegación del Gobierno para el Plan Nacional sobre Drogas. Ministerio del Interior. Observatorio Español sobre Drogas. Informe $n^{\circ}$ 4. 2000.

(5). Plan Europeo de Actuación sobre el Alcohol 2000-2005. (PEAA). Comité Regional de la OMS para Europa. Copenhague. 1998.

(6). García Más, MP. Evolución e intervención en la violencia familiar y alcoholismo. Socidrogalcohol. PND. Madrid. 1999.

(7). "Barómetro marzo 2001". Centro de Investigaciones Sociológicas (CIS). (No publicado). 